\title{
Logiques sociales et information télévisée
}

\section{Bernard Miège}

\section{(2) OpenEdition}

\section{Journals}

Édition électronique

URL : http://journals.openedition.org/edc/2845

DOI : $10.4000 /$ edc. 2845

ISSN : 2101-0366

Éditeur

Université Lille-3

\section{Édition imprimée}

Date de publication : 1 mai 1989

Pagination : 61-66

ISSN : 1270-6841

\section{Référence électronique}

Bernard Miège, «Logiques sociales et information télévisée », Études de communication [En ligne], 10 | 1989, mis en ligne le 11 février 2012, consulté le 19 avril 2019. URL : http:// journals.openedition.org/edc/2845; DOI : 10.4000/edc.2845

Ce document a été généré automatiquement le 19 avril 2019

(c) Tous droits réservés 


\section{Logiques sociales et information télévisée}

\section{Bernard Miège}

1 Si l'on applique les règles du « raisonnement » épistémologique aux travaux actuels portant sur la communication, aux recherches les plus diverses comme aux productions des penseurs de la modernité, on ne peut manquer d'être frappé par la diversité des approches et surtout par la très grande hétérogénéité des niveaux où elles se situent.

2 L'analyse de ces travaux amène, en effet, aux trois constats majeurs suivants; le champ de la communication serait :

- soit parcouru par des mouvements totalement erratiques, ou en tout cas relevant d'une analyse de la conjoncture (un nombre appréciable d'études s'épuisent ainsi à suivre des évolutions conjoncturelles et ne valent que pour des espaces de temps limités) ;

- soit redevable d'approches spécifiques employant des méthodologies et développant des problématiques largement mono-disciplinaires (c'est tout particulièrement le cas de travaux développés par des linguistes, par des politologues ou des psychologues sociaux);

- soit enfin l'objet et le lieu par excellence où appliquer des «théories générales » fondées sur un paradigme dominant, celui-ci leur paraissant capable de rendre compte de l'essentiel des phénomènes communicationnels aujourd'hui observables (cette façon de procéder est ancienne parmi les penseurs qui s'essaient à aborder le champ de la communication, mais elle renaît périodiquement; et dans la dernière période on la retrouve sous une forme manifeste chez Lucien Sfez et sa Critique de la communication, mais également malgré des apports certains chez Pierre Levy et sa Machine univers).

Il n'y aurait donc rien à redire. Et en quelque sorte, on serait condamné soit à décrire des mouvements conjoncturels, soit à appliquer des approches mono-disciplinaires spécifiques, soit à constater des effets de théories. Rares sont les productions récentes qui, d'une façon ou d'une autre, ne se positionnent pas ainsi, soit que leurs auteurs revendiquent explicitement l'une ou l'autre de ces orientations, soit que de facto ils s'en rapprochent. 
Comment se sortir de ce qu'il faut bien qualifier comme une impasse, le choix n'étant laissé qu'entre les descriptions conjoncturelles, les travaux étroitement spécialisés et les théories trop générales pour donner lieu à vérifications?

5 A notre avis, il y a place pour ce qui peut être appelé « problématiques transversales et partielles », celles-ci permettant à la fois de satisfaire le souci d'élaboration théorique (dont l'urgence est criante, en raison de l'effervescence présente et de la complexité des enjeux), et le souci d'approches empiriques (au sens fort et exigeant du terme), seul moyen de rendre compte de la complexité des situations de communication. Nous postulons donc que le progrès des connaissances viendra surtout de réflexions et de travaux, fondés sur des méthodologies inter-sciences, et traversant les champs couvrant la communication, sans prétendre envisager celle-ci dans sa totalité; dans cette perspective, l'élaboration théorique alliée avec et basée sur des observations empiriques menées aussi rigoureusement. que possible, doit amener à dégager des «règles de fonctionnement » ayant une suffisante stabilité temporelle et qui aident à expliquer, dans un champ spécifique, les relations qui s'établissent entre les acteurs sociaux concernés, relations qui nous apparaissent souvent totalement erratiques, car nous ne pouvons les « référer » à des éléments structurels.

6 Cette façon d'envisager l'approche de la communication s'inspire de ce qui a été tenté par Pierre Bourdieu à propos d'autres questions : tout particulièrement sa théorie des champs et la préoccupation qui est la sienne de mettre en évidence des "mouvements structurants/structurés ", nous paraissent d'un apport utile pour tenter d'apporter une réponse aux difficultés épistémologiques signalées précédemment.

Nous tenons cependant à marquer une différence sur un point essentiel : la théorie des champs opère le plus souvent un "découpage ", qui met sur le même plan des acteurs sociaux ne pesant pas du même poids (par exemple des producteurs et des consommateurs). En plus, elle se préoccupe insuffisamment de " positionner » les champs les uns par rapport aux autres. Or, cet objectif nous paraît être une condition indispensable dans le cas de la communication : que l'on se limite à des problématiques partielles relève, dans l'état actuel des connaissances, à la fois d'une nécessaire prudence méthodologique, et de la conscience de l'extrême difficulté à articuler des niveaux de l'analyse (par exemple: entre ce qui se rapporte à la communication médiatisée, à la communication médiatée ou à l'intercompréhension argumentative) ; mais, pour autant, il est indispensable d'envisager des approches transversales (par exemple allant des créateurs et des producteurs jusqu'aux usagers), ce qui implique clairement de situer les différents champs les uns par rapport aux autres. Ce type de problématique doit pouvoir déboucher sur des propositions diverses. Pour notre part, elle nous a amené à mettre en évidence ce que nous appelons logiques sociales. Sous cette dénomination qui ne correspond aucunement au sens que des auteurs comme Gabriel Tarde ou Mancur Olsen ont donné à la même expression, nous entendons identifier des mouvements de longue durée, portant aussi bien sur des processus de production que sur des articulations production/consommation, ou sur des mécanismes de formation des usages.

On doit admettre que les logiques sociales ne sont pas fixées définitivement et qu'elles se transforment régulièrement, surtout dans une période aussi explosive et ouverte que la période présente. L'important, en effet, est de ne pas considérer ces logiques sociales de façon déterminante ou mécanique, et de comprendre que c'est autour d'elles qu'à moyen terme, se développent, parfois en des sens opposés, les stratégies des acteurs sociaux; ceux-ci, dans leurs actions quotidiennes, ont la possibilité d'aller momentanément et 
même durablement, à leur encontre ; ce faisant, ils parviennent à déplacer les logiques dominantes, et même à s'en abstraire (tout particulièrement lorsqu'ils se placent dans des situations allternatives). On l'aura compris : avec elles, nous n'avons pas affaire à un modèle explicatif valable hic et nunc, s'imposant aux acteurs sociaux en ne leur laissant que de minces espaces de liberté. Car la communication est une « construction sociale » en cours à laquelle concourrent activement les différents acteurs sociaux concernés ; il en va de son insertion en profondeur dans les sociétés contemporaines.

On ajoutera que la mise en évidence de ces logiques sociales procède rarement d'une réflexion théorique abstraite; elles émergent le plus souvent au terme d'analyses et d'observations de pratiques et de politiques, car elles permettent notamment de dénouer des situations très imbriquées, où des enjeux de natures diverses se superposent et se concurrencent. A priori, c'est dans des situations de ce genre que le recours aux logiques sociales pour éclairer l'évolution très embrouillée des phénomènes communicationnels s'avère heuristique : nous allons en donner ci-après trois exemples, les deux derniers se rapportant plus directement au thème de ces Journées d'études.

\section{L'industrialisation des programmes informationnels et culturels.}

10 Le mieux est que je retrace brièvement ce qu'a été ma démarche. Dans un premier temps, mon intérêt s'est porté vers les industries culturelles, et tout spécialement vers l'édition de marchandises culturelles (livres, disques, entrées dans les salles de cinéma, vidéocassettes enregistrées, ...) ; ces produits édités consistent en la reproduction par des méthodes industrielles sur des supports matériels de productions originales, plus ou moins complexes, d'artistes ou d'intellectuels. On a pu, ainsi, mettre en évidence les règles de fonctionnement de ce qu'il est convenu d'appeler aujourd'hui le modèle éditorial, et en particulier celles-ci : caractère aléatoire des valeurs d'usage et difficultés de valorisation des produits; étalement des risques grâce au système du catalogue; rémunération des auteurs et interprètes par le moyen de droits d'auteurs et de reproduction ; maintien d'une petite et moyenne production à Côté de firmes dominantes surtout intéressées à contrôler la distribution; existence à côté du star system d'un vivier d'auteurs très mal rémunérés et prêts à s'employer à tous moments, etc...

11 Par la suite, j'en suis venu à élargir ma démarche et à considérer que deux autres modèles présidaient au fonctionnement de la plupart des autres industries culturelles et informationnelles, et devaient être mis en parallèle avec le modèle éditorial : d'abord le modèle de flot qui organise la production émanant de la radiodiffusion commerciale et dont l'objectif est d'obtenir, grâce à la diffusion programmée d'émissions distractives culturelles et informatives, la fidélisation d'auditoires pour les besoins du client principal des chaînes : les annonceurs publicitaires; ensuite le modèle de la presse écrite qui présente certains traits « empruntés » à l'un et à l'autre des modèles précédents, les entreprises de presse ayant à faire face à deux marchés, celui de leur lectorat et celui des annonceurs publicitaires. Il importe peu de présenter ici de façon détaillée les règles de fonctionnement propres à ces deux autres modèles (modalités de rémunération des travailleurs artistiques et intellectuels, organisation de la phase de conception des produits et du procès de production, relations avec les lecteurs et les auditeurs, procédés de fidélisation, organisation de la production facilitant la concentration, ...) ; l'important 
est de constater que les rapports entre ces trois modèles sont évolutifs, que chacun d'eux ne pèse pas du même poids, et surtout qu'une grande partie des enjeux parmi les plus marquants s'expliquent par un affrontement entre les trois modèles, qui se trouvent être en concurrence: et vis-à-vis des usagers, et dans les activités des artistes et des producteurs, et par rapport aux annonceurs. Ainsi a-t-on pu montrer que de nombreux conflits présents prenaient leur origine dans la montée du modèle de flot consécutive au développement des télévisions commerciales. La brutale impulsion donnée à ce modèle a eu des effets déstructurants dans l'ensemble de la production artistique, et dans presque toutes les industries culturelles, particulièrement dans le cinéma.

Les voies par lesquelles s'effectue l'industrialisation des activités culturelles et informationnelles peuvent difficilement échapper aux règles de fonctionnement des trois modèles. Bien plus, ces règles «marquent » d'une façon ou d'une autre, l'ensemble des pratiques, depuis le travail de conception ou de création jusqu'à la formation des usages ; on en donnera un seul exemple relatif à l'information télévisée : la « 5 », lors de sa deuxième « naissance » à l'automne 1986, a été amenée à reprendre telle quelle la formule du JT de 20 heures alors qu'elle aurait pu utiliser cette plage horaire très intéressante pour concurrencer les chaînes rivales par la diffusion de jeux attractifs et... de spots ; mais, ce faisant, elle s'est pliée aux règles de fidélisation de ses auditoires, dont on sait qu'ils restent particulièrement attachés à ce rite social quotidien forgé au cours des années soixante ; la simple logique commerciale l'aurait amenée à des choix différents.

\section{Le JT : un genre toujours en formation.}

13 Le JT va d'ailleurs nous permettre de délaisser le cas quelque peu emblématique des industries culturelles et informationnelles, pour mettre en oeuvre la problématique des « logiques sociales » dans un autre champ, celui de l'information à la télévision.

Une recherche collective récente (le JT: mise en scène de l'actualité à la télévision, INA-La Documentation Française, 1986) a été l'occasion de mener une réflexion, dont la position fondamentale consistait à refuser de choisir entre une sociologie ne tenant pas compte des caractéristiques formelles du Journal et une sémiologie oublieuse des enjeux sociaux qu'il recèle.

15 A partir de là, nous avons considéré qu'il n'était pas pertinent d'en suivre l'évolution en s'efforçant d'en apprécier l'information qui y est produite et diffusée (comme le font les spécialistes de l'analyse de contenu) : le travail journalistique, s'il n'est évidemment pas absent du JT, y est subordonné à d'autres exigences. Et paradoxalement, autant l'inscription du JT dans la société française est bien ancrée (on peut encore le tenir pour incontournable, indémodable et indépassable), autant le genre lui-même est encore hésitant : se présentant en permanence comme un objet d'évidence le JT emprunte à la fois à la presse écrite, aux techniques scénographiques et, plus faiblement, à la cinématographie; il n'est pas un genre fixé et tout indique qu'il doit régulièrement produire les conditions de sa reconnaissance et de sa légitimité sociales.

Telle est la logique qui paraît traverser les JT des chaînes de TV françaises et qui éclaire les multiples enjeux et les conflits, ouverts ou feutrés, qui s'y déroulent. Le JT : un genre hésitant parce qu'il concentre en lui certaines des marques essentielles de la société française contemporaine et qu'il est l'une des sources du lien social qu'il faut en permanence renouveler. 


\section{L'information aux prises avec la communication.} nettement les différences entre ce qui est de leur responsabilité et le monde de la communication, celle-ci étant le plus souvent assimilée à la publicité. Par la suite, ils ont hésité entre la préoccupation de prendre en charge cette nouvelle activité sociale et le respect des exigences ainsi que de la déontologie de l'activité journalistique.

Aujourd'hui, surtout dans un pays tel que la France où la presse écrite est dans une situation de faiblesse caractérisée, et où l'information télévisuelle porte encore nettement les traces, même atténuées, du fort contrôle politique qui a accompagné son développement, la question des rapports entre information et communication ne se pose plus dans les mêmes termes : une partie importante des organes d'information fonctionne en effet au rythme de la communication : à une presse faible et peu soucieuse d'enquêter font face désormais dans tous les secteurs de la vie économique, sociale et culturelle, des services dé communication (et de relations publiques) bien organisés et utilisant les compétences de professionnels qualifiés. Les journalistes sont sollicités plus qu'ils ne sollicitent, les sujets de leurs papiers leur sont souvent proposés, et dans la plupart des cas on leur remet des « dossiers de presse » fort bien préparés dans lesquels ils puisent d'autant plus facilement qu'ils n'ont guère le temps, à l'exception de ceux appartenant aux grands journaux, de préparer longuement leurs articles ou leurs sujets. Tendanciellement c'est surtout la presse spécialisée, la presse quotidienne régionale et les stations régionales de FR 3 qui semblent le moins résister aux entreprises des spécialistes de la communication.

19 La connivence qui s'est installée entre les organes d'information et les professionnels de la communication (à qui d'ailleurs on ne peut imputer le déséquilibre constaté), joue de plus en plus nettement en défaveur de l'information, en raison bien sûr des faiblesses structurelles de la presse mais aussi en raison de l'absence de traditions d'indépendance suffisamment fermes du journalisme à l'égard des « pouvoirs ».

D'autres aspects de ces relations dissymétriques pourraient être mis en évidence ; mais nous en resterons là car notre but ici était de montrer qu'une logique de plus en plus identifiée par les acteurs eux-mêmes, imprègne l'ensemble des relations entre l'information et la communication.

21 La problématique des logiques sociales n'est que l'une de ces problématiques transversales et partielles qui nous paraissent de nature à faire progresser la recherche en communication beaucoup plus sûrement que les théories générales qui fleurissent régulièrement et entendent, à l'aide d'un unique paradigme, traiter de la complexité de la communication. Cette voie de la recherche, dont nous avons esquissé les orientations à partir d'exemples portant sur l'information, est riche de possibilités; elle peut en particulier aider à dépasser les conséquences néfastes des stériles oppositions macro/ micro, structures/interactions voire structures sociales/activité langagière. 


\section{RÉSUMÉS}

Faisant le constat d'un problème épistémologique auquel font face les sciences de la communication (séparation de la théorie et de la pratique, monodisciplinarité, analyses conjoncturelles) l'auteur propose une nouvelle orientation interdisciplinaire qui pourraient à la fois satisfaire les besoins théoriques et empiriques des chercheurs. S'inspirant de la théorie des champs de Bourdieu définit prudemment la notion de logique sociale qui permet d'analyser les processus de production comme les usages. Ce propos théorique est illustré par trois exemples sur l'industrie audiovisuelle et l'information télévisée.

\section{INDEX}

Keywords : social logic, model, television news, information, journalism, culture industry, epistemology of communication sciences

Mots-clés : logique sociale, journal télévisé, information, journalisme, industrie culturelle, épistémologie de la communication

\section{AUTEUR}

\section{BERNARD MIÈGE}

Bernard Miège, GRESEC, Université Stendhal de Grenoble 